\title{
Design of Ultrasonic Linear Array Transducer
}

\author{
Gandole Y.B. ${ }^{1}$, Rodge S.A. ${ }^{2}$ \\ (Departmentof Electronics, Adarsha Science J.B.Arts and Birla Commerce Mahavidyalaya, Dhamangaon Rly - \\ 444709, India)
}

\begin{abstract}
Ultrasonic arrays are used in many applications including medical imaging. In this specific case is important to achieve precise information about the magnitude and position of the peak pressure and intensity produced by the probe. This paper presents the designof linear array transducers for ultrasonic measurements Keywords: Ultrasonic, Linear array, Transducer,medical imaging.
\end{abstract}

\section{Introduction}

In the biomedical field, the systems for images processing are very important calling for new techniques, much more advanced and performing than they used to be, in order to provide a correct analysis and diagnosis.Ultrasound, widely used in many areas of medicine, provides a safe and efficient means for diagnosis and therapy. There are considerable efforts in designing transducers and determining the characteristics of the emitted field. To simulate the pressure response from transducers, a model which can predict the volt-to-surface acceleration conversion of multilayered transducers is needed. Methods for modeling piezoelectric transducers are well known in literature.[1-6]. Most of these models are based on electrical equivalent circuits benefiting from transmission line theory to represent the electromechanical coupling and acoustic wave propagation, and others rely on deriving impedance matrices for describing the transducer behavior. All of these methods have their advantage and disadvantages depending on the application of use. There are considerable efforts in designing transducers and determining the characteristics of the emitted field. Field II program [7], developed by J.A. Jensen, can simulate all kinds of ultrasound transducers using linear acoustics and it utilizes the Tupholme-Stepanishen method for calculating spatial impulse responses. The program is capable of calculating the fields for both the pulsed and continuous wave case for a large number of different transducers and allows visualization of simulating transducers. The calculation of the spatial impulse response assumes linearity [8] and any complex-shaped transducer can therefore be divided into smaller apertures and the response can be found by adding the responses from the sub-apertures.

\section{Spatial Impulse Theory}

The pressure field generated by the aperture is found by the Rayleigh integral [9]

$p\left(\vec{r}_{1}, \mathrm{t}\right)=\frac{\rho_{0}}{2 \pi} \int_{s} \frac{\partial v_{n}\left(\overrightarrow{r_{2}} \cdot \mathrm{t}-\frac{\left|\overrightarrow{r_{1}}-\overrightarrow{r_{2}}\right|}{c}\right)}{\left|\overrightarrow{r_{1}}-\overrightarrow{r_{2}}\right|} d s$

where the field point is denoted by $\vec{r}_{1}$ and the aperture by $\vec{r}_{2}$, is the velocity normal to the transducer surface. Using the velocity potential, and assume that the surface velocity is uniform over the aperture making it independent of $\vec{r}_{2}$, then: where the field point is denoted by $\vec{r}_{1}$ and the aperture by $\vec{r}_{2}$, is the velocity normal to the transducer surface. Using the velocity potential, and assume that the surface

velocity is uniform over the aperture making it independent of $\vec{r}_{2}$, then:

$\Psi\left(\overrightarrow{r_{1}}, \mathrm{t}\right)=v_{n}(t) * \int_{s} \frac{\partial\left(\mathrm{t}-\frac{\left|\overrightarrow{r_{1}}-\overrightarrow{r_{2}}\right|}{c}\right)}{2 \pi\left|\overrightarrow{r_{1}}-\overrightarrow{r_{2}}\right|}$

where * denotes convolution in time. The integral in this equation

$h\left(\vec{r}_{1}, \mathrm{t}\right)=\int_{s} \frac{\partial\left(\mathrm{t}-\frac{\left|\vec{r}_{1}-\vec{r}_{2}\right|}{c}\right)}{2 \pi\left|\vec{r}_{1}-\vec{r}_{2}\right|}$ 
represent the spatial impulse response. The continuous wave field can be found from the Fourier transform of

$$
p\left(\overrightarrow{r_{1}}, \mathrm{t}\right)=\rho_{0} \frac{\partial v(t)}{\partial t} * h\left(\vec{r}_{1}, \mathrm{t}\right)
$$

The impulse response includes the excitation convolved with both the transducers electro-mechanical impulse response in transmit and receive. The final signal for a collection of scatters is calculated as a linear sum over all signals from the different scatters [10].

\section{Linear Array Transducer}

The linear array is the fundamental type of multi-element transducer and it scans the region of interest by exciting the elements situated over the region. The field is focused on the region by introducing time delay in the excitation of the concerned individual elements, so initially concave beam is emitted. Here a 16 element linear array transducer is designed using FIELD-II program as shown in the Fig.1, height, width and kerf of individual element are taken as $5 \mathrm{~mm}, 0.2 \mathrm{~mm}$ and $0.02 \mathrm{~mm}$ respectively. The transducer is situated at the center of the coordinate system. The electronic focusing is incorporated to achieve focal length of $\mathbf{3 0} \mathrm{mm}$ from the center of transducer. For the above linear array transducer, an excitation signal of two cycles of sinusoidal pulses is given in Fig. 2(a). The impulse response pattern obtained for each element is shown in Fig. 2(b).
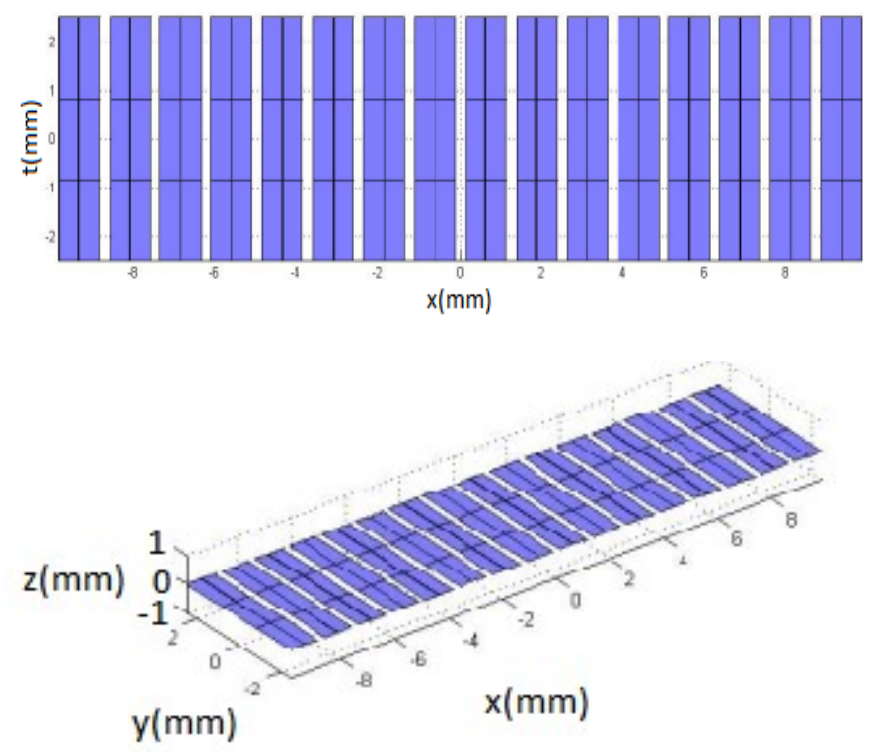

Fig. 1 Design of linear array transducer (Height $=5 \mathrm{~mm}$, Width $=0.25 \mathrm{~mm}$, Kerf $=0.02 \mathrm{~mm}$ )

For this specified linear array, acoustic field generated is propagated through human body tissues and is observed at a focal distance i.e. $(0,0,30)$ whose plot is shown in Fig. 4. Fig.

4(a) shows the field generated while Fig. 4(b) shows the pressure profile at focal distance. Similarly Fig. 5 illustrates the lateral beam pattern generated by the assumed linear array transducer. The other simulation parameters chosen are central transducer frequency $(\mathrm{f} 0)=5 \mathrm{MHz}$, acoustic speed $(\mathrm{c} 0)=1540 \mathrm{~m} / \mathrm{s}$, fractional bandwith $50 \%$, center frequency of excitation pulse $=7.5 \mathrm{MHz}$, Number of cycle $=5$.

\section{Result And Discussion}

The calculation of the impulse response is facilitated by projecting the field point onto the plane of the aperture. In this way, the problem became two-dimensional and the field point is given as a $(x, y)$ coordinate set and a height $\mathrm{z}$ above the plane. The spatial impulse response is, thus, determined by the relative length of the part of the arc that intersects the aperture. Thereby it is the crossing of the projected ultrasonic waves with the edges of the aperture that determines the spatial impulse responses as a function of time.

By using FIELD II program were created three linear arrays with the same characteristics, but with different number of elements: 16 elements, 32 elements and 64 elements. Respectively. For the simulations the transducer center frequency was set to $f 0=5 \mathrm{MHz}$. The speed of sound in tissue is $c=f 0=1540 \mathrm{~m} / \mathrm{s}$, which gives a wavelength of $\mathrm{mm}$. The sampling frequency used was $f_{s}=100 \mathrm{MHz}$. The elements had a width and height of $0.25 \mathrm{~mm}$ and $5 \mathrm{~mm}$ respectively. The focal-point was set to $30 \mathrm{~mm}$.

Then the normalized spatial impulse response for this aperture was calculated and plotted by time. Figures 2, 3, 4 and 5 shows the normalized spatial impulse response, Transducer Pressure field, Lateral beam 
plot and Transmitted Pressure Field in the focal plan for 16 elements. Figures 6, 7, 8 and 9 shows the normalized spatial impulse response, Transducer Pressure field, Lateral beam plot and Transmitted Pressure Field in the focal plan for 32 elements. Figures 10,11,12 and 13 shows the normalized spatial impulse response, Transducer Pressure field, Lateral beam plot and Transmitted Pressure Field in the focal plan for 64 elements.

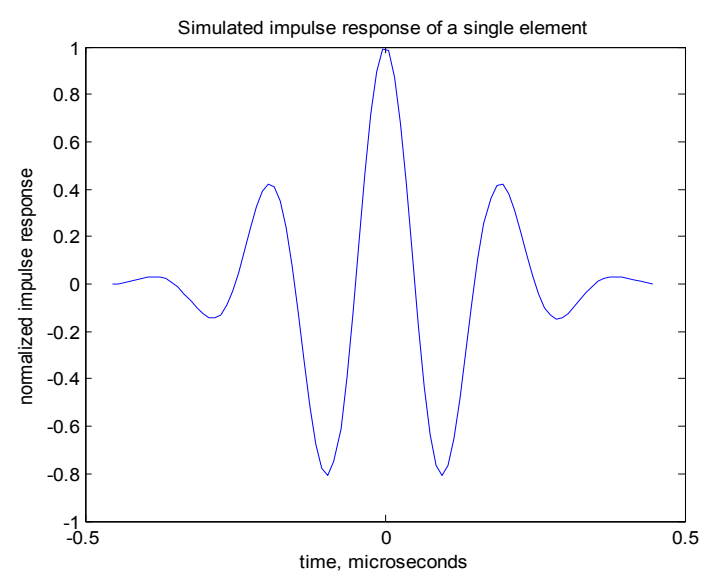

Fig. 2: Normalized impulse response of a single element.

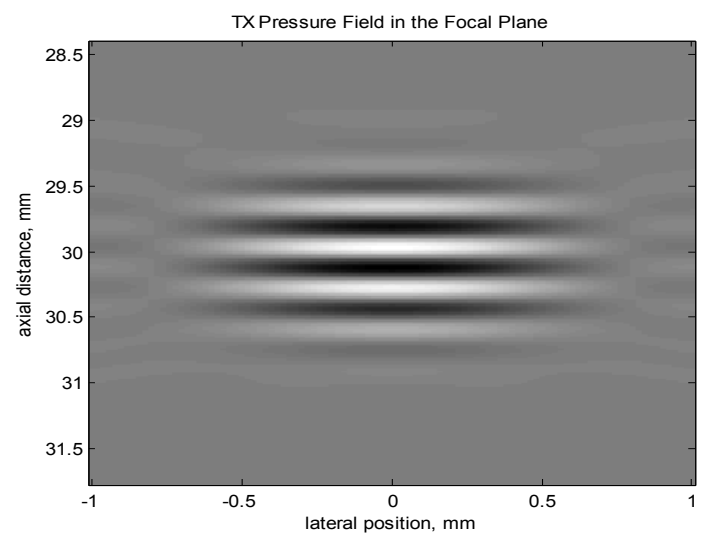

Fig. 3: Transducer Pressure field for 16 elements.

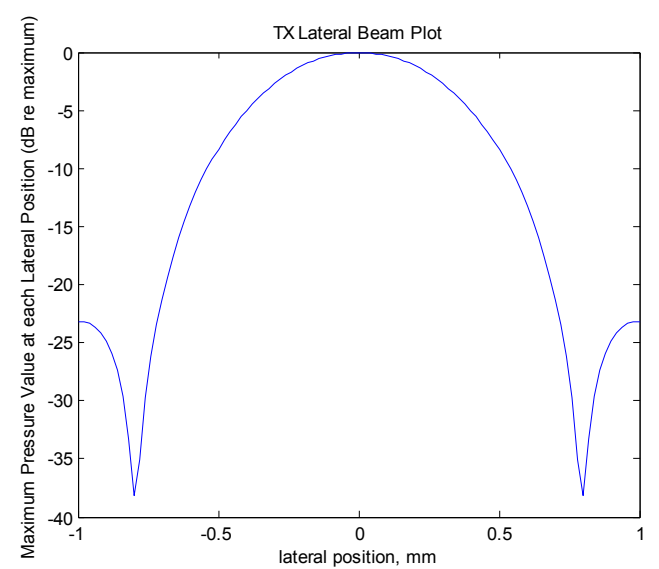

Fig. 4: Lateral beam plot of 16 elements 


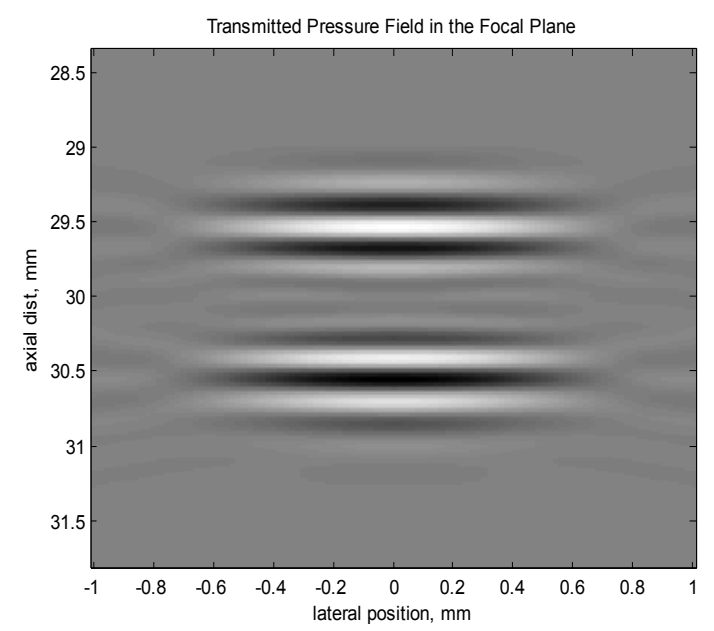

Fig. 5: Transmitted Pressure Field in the focal plan for 16 elements.

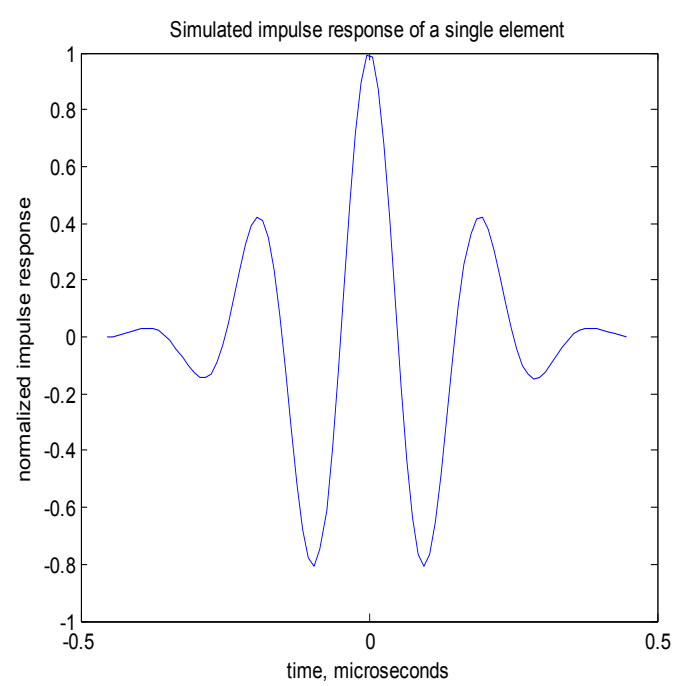

Fig. 6: Normalized impulse response of a32 elements.

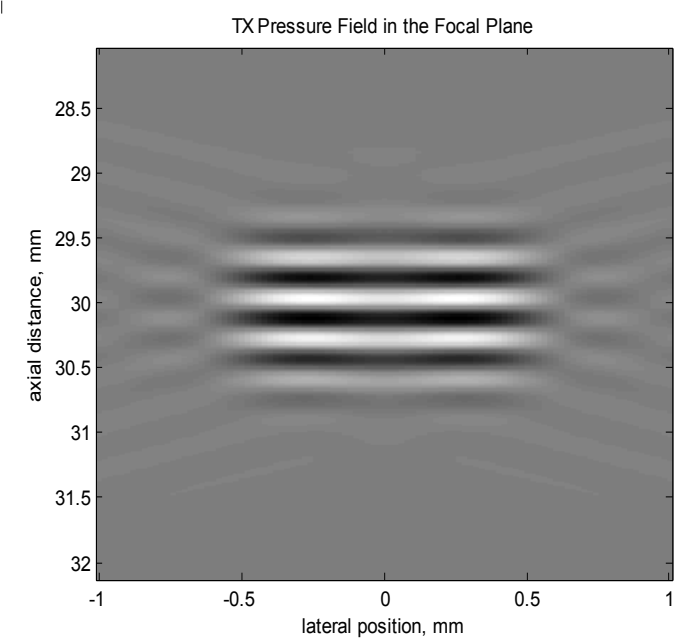

Fig. 7: Transducer Pressure field for 32 elements. 


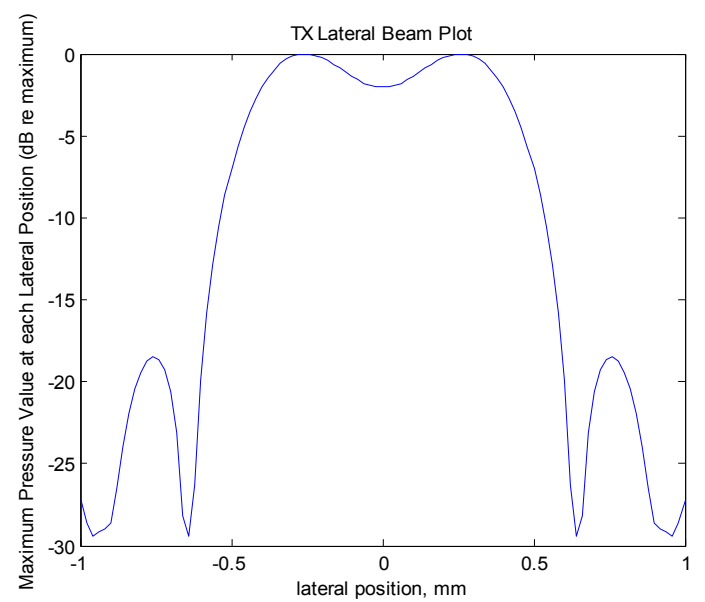

Fig. 8: Lateral beam plot of 32 elements.

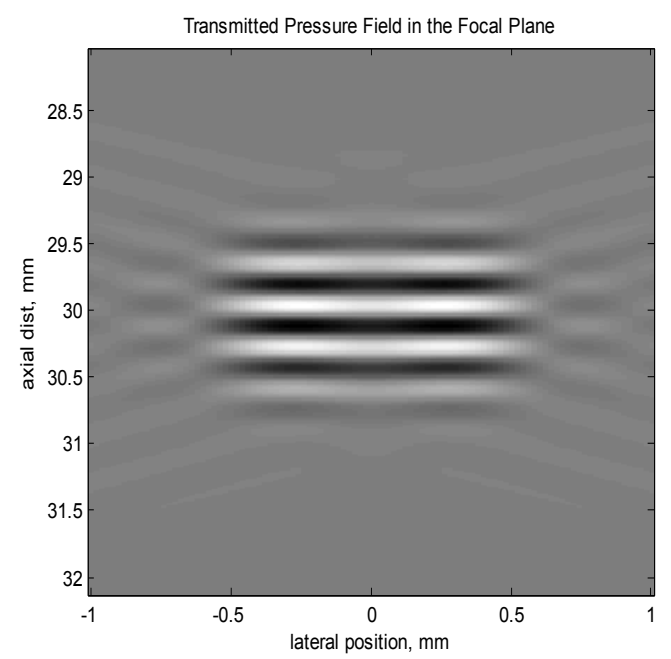

Fig. 9: Transmitted Pressure Field in the focal plan for 32 elements.

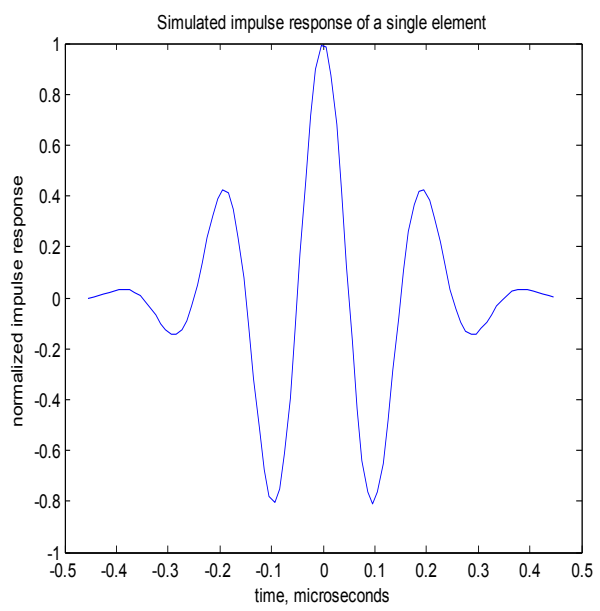

Fig. 10: Normalized impulse response of a64 elements. 


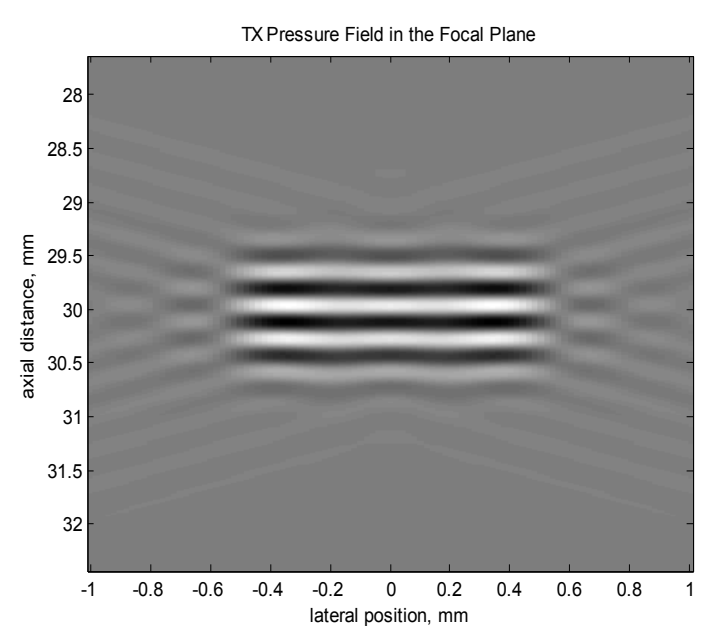

Fig. 11: Transducer Pressure field for 64 elements.

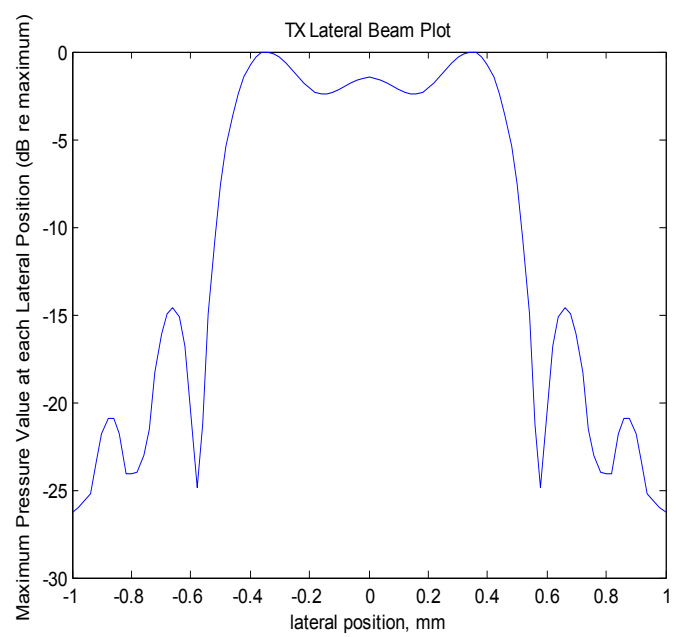

Fig. 12: Lateral beam plot of 64 elements.

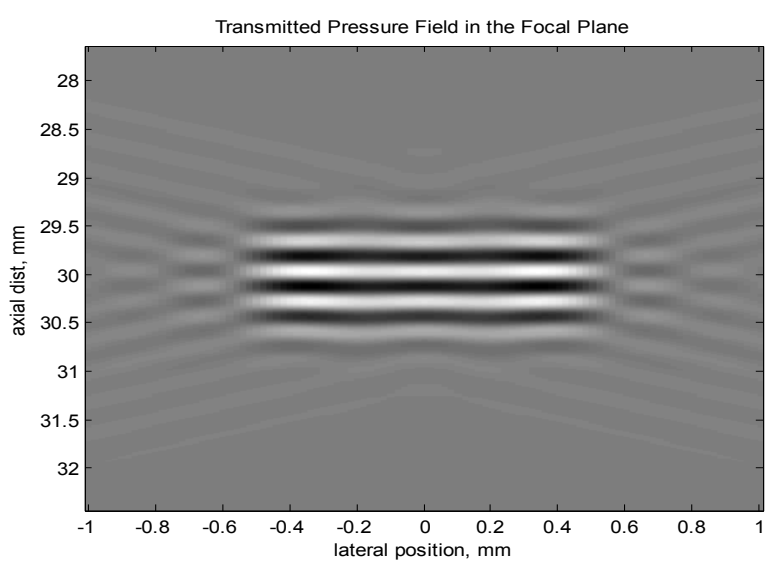

Fig. 13: Transmitted Pressure Field in the focal plan for 64 elements.

\section{Conclusion}

The paper attempts to present a coherent analysis of the focusing strategies for 2-D array transducer design and properties, based on linear acoustics. The delays on the individual transducer elements and their relative weight or apodization are changed continuously as a function of depth. This yields near perfect focused images for all depths and has increased the contrast in the displayed image, thus, benefitting the diagnostic value of ultrasonic imaging. 


\section{References}

[1] M. Redwood, “Transient performance of a piezoelectric transducer,” J. Acoust. Soc. Am. 33, 527-536,1961.

[2] E. K. Sittig, "Transmission parameters of thickness-driven piezoelectric transducers arranged in multilayer configurations," IEEE Trans. Sonics Ultrason. SU-14, 167-174, 1967.

[3] R. Krimholtz, D. Leedom, and G. Matthaci, "New equivalent circuits for elementary piezoelectric transducers," Electron. Lett. 6, 398-399, 1970.

[4] T. R. Meeker, "Thickness mode piezoelectric transducers," Ultrasonics 10, 26-36,1972.

[5] P. Marchal, F. Levarssort, L.-P. Tran-Huu-Hue, and M. Lethiecq, "Effects of acoustical properties of a lens on the pulse-echo response of a single element transducer," IEEE International Ultrasonics, Ferroelectrics, and Frequency Control Joint 50th Anniversary Conference, pp. 1651-1654,2004..

[6] P. Maréchal, F. Levarssort, L.-P. Tran-Huu-Hue, and M. Lethiecq, "Lensfocused transducer modeling using an extended KLM model," Ultrasonics 46, 155-167, 2007.

[7] JENSEN, J.A., Field: A program for simulating ultrasound systems, 10th Nordic-Baltic Conference on Biomedical Imaging, in: Medical \& Biological Engineering \& Computing, 1996, 34, Supplement 1, 351-353.

[8] JENSEN, J.A, A New Approach to Calculating Spatial Impulse Responses, IEEE International Ultrasonic Symposium, Toronto, Canada, 1997

[9] Jensen, J.A, A New Approach to Calculating Spatial Impulse Responses, IEEE International Ultrasonic Symposium, Toronto, Canada, 1997

[10] Jensen, J.A., N.B. Svendsen, Simulation of advanced ultrasound systems using field II, Biomedical Imaging: Nano to Macro, 2004. IEEE International Symposiumon, 15-18 April 2004 Page(s):636-639 Vol.1 